\title{
Understanding Stormwater Pipe Deterioration Through Data Mining
}

\author{
R. Richard Harvey and Edward A. McBean
}

\begin{abstract}
Stormwater pipe condition is commonly assessed using closed circuit television (CCTV) inspection, a task which is both expensive and time consuming. Consequently, most municipalities have been limiting their inspections to small portions of their stormwater systems. A data mining procedure using a powerful classification tree methodology is developed to extract asset condition information related to stormwater pipe integrity. A case study illustrates the process of developing classification trees using a dataset of pipe condition obtained after inspecting one third of the stormwater pipes in Guelph, Ontario. The classification tree illustrates the influence of construction year, diameter, length and slope on pipe condition in an easily interpretable format. An overall success rate of $71 \%$ (301/425 instances of pipe condition correctly classified in a stratified test set) indicates the utility of the model when predicting the condition of the remaining two thirds of the pipes in the stormwater system that have not yet been inspected.
\end{abstract}

Keywords: monitoring, storm water, infrastructure, modeling, data mining.

\section{Introduction}

\subsection{The Threat Posed by Deteriorating Storm- water Pipes}

Effective stormwater system management is a challenging task, particularly for older municipalities dealing with pipes that have been in operation for $>50 \mathrm{y}$. The replacement value of the $23 \%$ of Canadian stormwater pipes currently estimated to be in fair to very poor condition is an estimated $\$ 15.8$ billion, or $\$ 1270 /$ household (CIRP 2012). Many of these pipes cannot effectively transport stormwater runoff from impervious surfaces to oceans, lakes or artificial ponds as originally intended. An estimated one in ten stormwater pipes are unfit for handling rainfall during extreme weather events and the resulting floods can have significant financial consequences (CIRP 2012). As an example, 150 $\mathrm{mm}$ rainfall over a $3 \mathrm{~h}$ period in Toronto, Ontario in 2005 flooded basements, washed away roads, overloaded treatment plants and destroyed water, wastewater and stormwater pipes. The storm was responsible for more than $\$ 500$ million in damages and is considered to be the most expensive natural disaster to ever occur in Ontario (Kessler 2011).

The infiltration of groundwater into stormwater pipes through defective joints, cracks and holes accelerates the process of pipe aging and increases the failure probability of adjacent infrastructure due to the flushing of backfill material around the leak (Karpf and Krebs 2011). The resulting failure of overlying roadways can be unexpected, as was the sinkhole caused by the failure of a 50 year old stormwater pipe in Ottawa, Ontario that swallowed the car of a motorist and disrupted traffic flow along a major highway for weeks (Hurley 2012). The failure of a 120 year old brick stormwater pipe in Baltimore, Maryland created multiple sinkholes along a busy street near Johns Hopkins hospital. Repairs to the pipe and other surrounding infrastructure were expected to last several months, with an estimated cost of $\$ 7$ million (Reutter 2012).

An indication of further issues associated with stormwater pipe integrity exists because these pipes often carry high levels of fecal indicator bacteria and are a major contributor to poor surface water quality in urban areas (USEPA 2009). Although fecal matter in the stormwater system has traditionally been assumed to originate from the excrement of domestic pets and wildlife, growing evidence suggests wastewater leaking from defective sanitary sewers is another major contributor to stormwater pipe fecal contamination. The passage of untreated wastewater from defective sanitary sewers into structurally unsound stormwater pipes poses a potentially serious threat to the environment. Stormwater outfalls studied in Milwaukee, Wisconsin were found to be highly contaminated with sewage, suggesting that the pipes were major contributors to poor beach, river and stream water quality (Sauer et al. 2011). Field experiments carried out in California indicate sanitary sewer leaks are responsible for severe sewage pollution in stormwater pipes, even during dry weather flow (Sercu et al. 2011). An estimated 30\% of Canadian sanitary sewers are in fair to very poor condition (CIRP 2012) and it can be reasonably assumed that Canadian urban stormwater systems are experiencing levels of sewage contamination similar to those observed in the United States.

Sanitary sewers carry a wide range of chemicals and endocrine disruptors capable of causing long term damage to environmental and human health if they pass untreated into stormwater pipes and thence to surface water. Endocrine disruptors are particularly dangerous as these chemicals interfere with the natural hormones in the body responsible for reproduction, 
development and behavior (Holtz 2006). Kolpin et al. (2002) identified a variety of emerging contaminants and other wasteassociated chemicals in $80 \%$ of 139 rivers and streams surveyed across the United States.

\subsection{Inspecting the Threat}

Many municipalities have recognized the threat posed by deteriorating stormwater pipes and are actively determining pipe condition using CCTV. This non-destructive inspection technique is conducted by recording the internal condition of a pipeline using a small camera mounted on a robot. An operator controls the camera from the surface and reviews the transmitted video on a display screen. The operator is trained to identify defects (e.g. cracks and collapsed sections) and to set defect severity scores according to systems designed to minimize subjective evaluation of the camera footage. One of the most commonly used rating systems is the Water Research Center Manual of Sewer System Classification (WRc MSSC). According to this system each inspected pipe is assigned an ordinal internal condition grade (ICG) from 1 (no structural defects) to 5 (collapsed section or collapse imminent) based on defect severity.

Although CCTV inspections provide insight into pipe condition, they are time consuming and expensive. As a result, most municipalities are limited to performing inspections of small portions of their entire stormwater network. Advanced asset management processes capable of extracting valuable information pipe deterioration from existing condition records are therefore required to enhance the value of their inspection efforts.

\subsection{Modeling Pipe Deterioration}

Proactive stormwater management can be supported by the use of deterioration models that use information gained from inspection to predict the current and future condition of pipes. Such models are still in their infancy, as models devoted exclusively to stormwater pipes have received significantly less attention than those for watermains and sanitary sewers (Ana and Bauwens 2010).

Micevski et al. (2002) used Markov models to predict changes in structural condition for stormwater pipes grouped according to factors such as diameter and length in Newcastle, Australia. While their models performed well at the network level, they were not well suited to predicting the condition of individual pipes. Tran (2007) compared the performance of statistical Markov and ordinal regression models against neural networks when predicting stormwater pipe condition in Dandenong, Australia. These models were developed using CCTV data collected from 417 pipe segments, representing $27 \mathrm{~km}$ pipe or $2.2 \%$ of the total length of the stormwater system. Neural networks were better suited for predicting structural condition (ordinal values ranging from 1 to 3), although they suffered from a number of serious limitations related to model development (e.g. time consuming process of defining the optimal network architecture). Tran and $\mathrm{Ng}$ (2010) revisited the Dandenong dataset and support vector machines were found to be capable of marginally outperforming neural networks when predicting stormwater pipe condition. The overall success rate for instances in their dataset reserved for testing performance was $76 \%$ for the support vector machine model, compared to $74 \%$ for the neural network.

While predictions of condition provided by support vector machines and neural network models are useful for asset management purposes, these models are essentially black boxes. As such, information learned about pipe deterioration is deeply embedded within the models and is not easily interpreted. These black boxes do not explicitly indicate the relationship between attributes and condition and cannot answer questions along the lines of "What pipe diameters have a greater likelihood of being in poor structural condition?" Alternatively, pipe deterioration models can be developed using classification tree algorithms so that knowledge extracted from inspection datasets is represented in an easily understood format.

Classification tree algorithms are among the most powerful approaches for knowledge discovery and data mining (Rokach and Maimon 2007). They produce interpretable white box models, are robust to missing values and are insensitive to skewed data distributions, so making data preparation for modeling less cumbersome than with neural networks and support vector machines (Berry and Linoff 2004). As a result, classification trees are very popular within the data mining community and are commonly used in the fields of remote sensing (Tooke et al. 2009), finance (Sahin and Duman 2011), marketing (Moore and Carpenter 2010) and medicine (Podgorelec et al. 2002).

\section{Data Mining Methodology}

\subsection{The C5.0 System}

The C5.0 system (Quinlan 2012) can be used to develop classification tree models capable of predicting individual stormwater pipe condition. The C5.0 system is the successor of the C4.5 system first developed by Australian computer scientist Ross Quinlan (QuinIan 1993). This new system is significantly faster than C4.5 and can achieve similar results but with smaller, easier to interpret trees. The C5.0 system constructs classification trees in two phases:

1. Grow the initial classification tree using a divide-andconquer approach: training instances are split using an attribute that provides the maximum information gain. One branch is created for each possible value of the specified attribute and the dataset is split into two subsets. This process continues for each subset defined by the first split until the subsets cannot be split any further (Witten et al. 2011, chap. 4); and

2. Prune the tree: remove splits that make an insignificant contribution to the tree's predictive power (IBM 2011).

The developed classification tree provides a map of the classification process. Instances are classified by starting at the 
top(root) node of the tree containing the entire training dataset. The attributes specified by the root node are evaluated and a path is then followed downwards corresponding to the specified attribute splits. Each instance belongs to only one leaf in the tree and the distribution of instances at the leaf determines the resulting classification. By default, a probability threshold of 0.50 is used so that instances are labeled with the most probable class (McCarthy et al. 2005).

\section{Case Study}

\subsection{CCTV Inspections of Guelph's Stormwater Pipes}

Guelph is located in southwestern Ontario and has a population of 120000 . This fifth fastest growing city in Canada relies on a $432 \mathrm{~km}$ stormwater network, consisting of 8073 pipes constructed from 1903 to 2011 . The stormwater network has grown at a rate of $100 \mathrm{~km}$ every 15 years since 1945 (Figure 1).

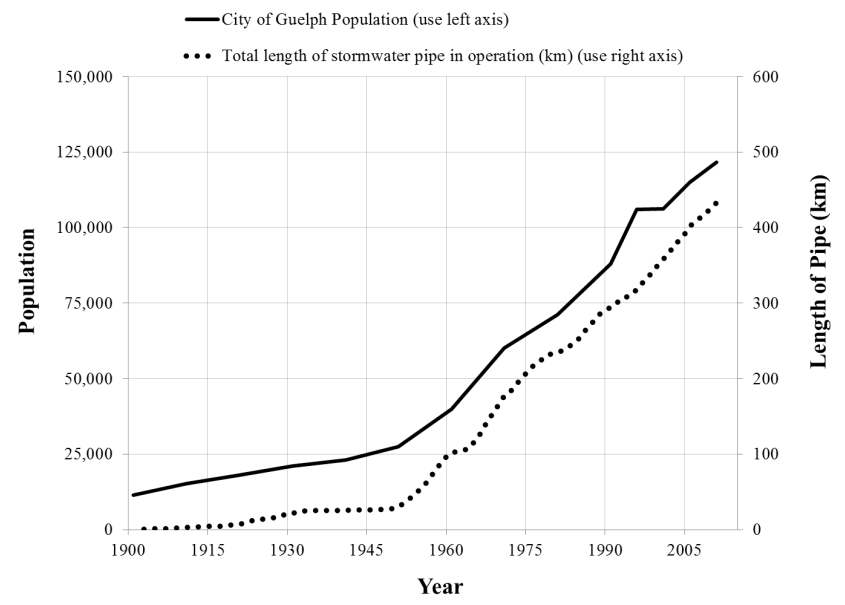

Figure 1 Length of Guelph stormwater system by year.

The average stormwater pipe section is 33 y old and $22 \%$ of the pipes have been in operation for $>50 \mathrm{y}$. The average pipe length is $54 \mathrm{~m}$, average diameter is $540 \mathrm{~mm}$, and average pipe slope is $1.45 \%$. Stormwater pipes are predominantly made of concrete (2 853), extra strength concrete (756) and reinforced concrete (2 184). Other pipe materials include PVC (491), polyethylene (162), corrugated steel (168), miscellaneous (55) and unknown (1 421).

CCTV inspections of approximately one third of Guelph's stormwater pipes were carried out from 2008 to 2011 (Figure 2). Defects inside each pipe were reviewed using the third edition of the Water Research Centre Manual of Sewer Condition Classification (WRc MSCC). These defects were then assigned a severity score using the fourth edition of the Water Research Centre Sewerage Rehabilitation Manual (WRc SRM). An internal condition grade (ICG) was then assigned to each pipe based on severity score thresholds established in the WRc SRM.

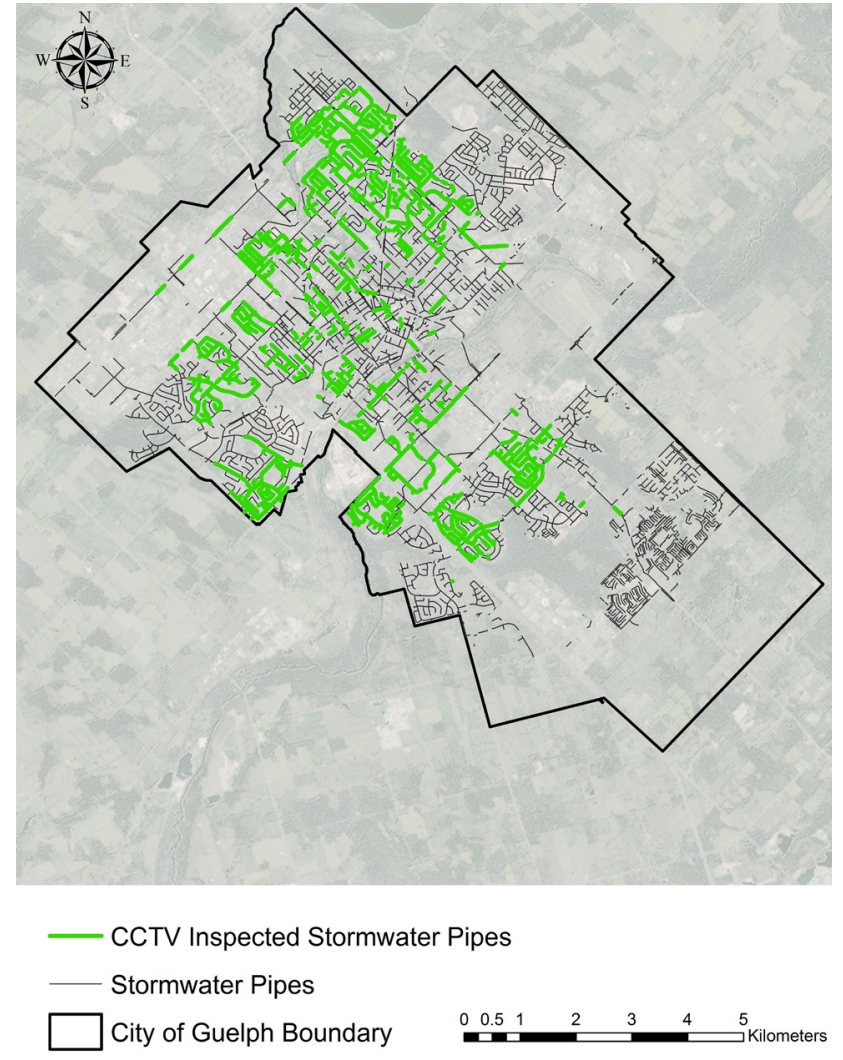

Figure 2 Inspected stormwater pipes.

A dataset of 1699 instances of stormwater pipe condition (total inspection length $107 \mathrm{~km}$ ) was used for model development. A total of 759 inspections were excluded from data mining for the following reasons:

- 552 inspected pipes were of an unknown construction material;

- Recorded material of construction for 179 inspected pipes did not match the material of construction observed during inspection;

- 23 PVC pipe inspections with construction dates ranging from 1985 to 2003 (every PVC pipe that was inspected had an ICG of 1); and

- five vitrified clay pipe inspections.

Of the 1699 inspected pipes used for model development, 1073 were made of concrete, 287 were extra strength concrete and 339 were reinforced concrete. Condition grades associated with each pipe material are presented in Table 1.

Table 1 Internal condition grades by pipe material.

\begin{tabular}{lcccccccc}
\hline \multicolumn{1}{c}{ Material } & $\begin{array}{c}\text { Age Range } \\
\text { (years) }\end{array}$ & \multicolumn{4}{c}{ Internal Cond. Grade (ICG) } & \multicolumn{3}{c}{ Structural Condition } \\
& 1 & 2 & 3 & 4 & 5 & ICG 1-2 & ICG 3-5 \\
\hline Concrete & $14-103$ & 397 & 232 & 342 & 78 & 24 & 629 & 444 \\
ES Concrete & $22-59$ & 113 & 91 & 68 & 14 & 1 & 204 & 83 \\
Reinf. Concrete & $5-56$ & 261 & 50 & 24 & 2 & 2 & 311 & 28 \\
Total & & 771 & 373 & 434 & 94 & 27 & 1144 & 555 \\
\hline
\end{tabular}


Guelph considers pipes with an ICG of 1 or 2 to be in good condition; those with an ICG of 3, 4 or 5 are in poor condition as they have already failed (holes, broken sections or collapse) or have a reasonable risk of failure in the near future. This creates the following binary modeling task: To develop a classification tree model that is easy to interpret and capable of reliably predicting whether individual pipes belong to the good or poor condition class.

Pipe-specific attributes considered for model development were material of construction, year of construction, diameter, length, slope, upstream invert elevation and downstream invert elevation (Table 2). These attributes were obtained using an ArcGIS stormwater inventory database maintained by the municipality. Burial depth, soil conditions around the pipe, and wall thickness were not included in the database and therefore could not be used as inputs for the predictive model. While the database contained information on the strength of the concrete pipes (e.g. class III reinforced concrete), this information was largely incomplete and was considered unfit for modeling purposes.

Table 2 Attributes used for model development.

\begin{tabular}{lll}
\hline \multicolumn{1}{c}{ Attribute } & Type & \multicolumn{1}{c}{ Description } \\
\hline Material & Nominal & Concrete, extra-strength concrete or reinforced concrete \\
Construction Year & Numeric & $1910-2008$ \\
Diameter & Numeric & $200 \mathrm{~mm}-2100 \mathrm{~mm}$ \\
Length & Numeric & $5 \mathrm{~m}-275 \mathrm{~m}$ \\
Slope & Numeric & $0.01 \mathrm{~m}-5.58 \mathrm{~m}$ rise for every $100 \mathrm{~m}$ run \\
Upstream invert elevation & Numeric & $305 \mathrm{~m}-356 \mathrm{~m}$ above mean sea level \\
Downstream invert elevation & Numeric & $305 \mathrm{~m}-356 \mathrm{~m}$ above mean sea level \\
\hline Attributes not available for analysis: burial depth, soil condition and wall thickness. \\
Attributes not suitable for modeling purposes: material strength.
\end{tabular}

The original dataset (1 699 instances of pipe condition: 1144 in good condition and 555 in poor condition) was randomly divided into a training set (75\% of all instances: 858 good, 416 poor) and a stratified test set (25\% of all instances: 286 good, 139 poor). The inspection dataset is imbalanced, in that there are significantly more pipes in one condition class than the other. The stratified sampling strategy of proportional allocation ensures that the predictive performance of any developed tree is properly evaluated as the class distribution in the test set is the same as the distribution in the original dataset.

Attributes were not transformed to a standardized scale as classification trees are largely unaffected by differences in scale between numeric attributes, and any transformation would have negatively impacted the resulting interpretability of any developed tree model.

\subsection{Evaluating Predictive Performance}

Predictive performance on a binary classification task is typically evaluated using the confusion matrix shown in Table 3 (with poor pipes being designated the positive class of interest). True positives and true negatives represent correctly classified stormwaterpipes. False positives occur when a pipe that was actually in good condition is incorrectly predicted to be poor. False negatives occur when a poor pipe is misclassified as being good.

Table 3 Condition prediction outcomes.

\begin{tabular}{lcc}
\hline \multicolumn{1}{c}{ Actual Condition } & Poor (ICG 3 - 5) & Good (ICG 1 - 2) \\
\hline Poor (ICG 3 -5) & True positives (TP) & False negatives (FN) \\
Good (ICG 1 - 2) & False positives (FP) & True negatives (TN) \\
\hline Note: classification results are often presented using a format known as a confusion matrix. The \\
number of instances/examples for which the actual pipe condition observed via CCTV inspection is \\
the column and the condition predicted by the classification tree is the row.
\end{tabular}

The confusion matrix can be used to determine the overall success rate (OSR) and the false negative rate (FNR) for both the the training set and stratified test set:

$$
\begin{aligned}
& O S R=\frac{\text { correctly classified instances }}{\text { total number of instances }} \\
& F N R=\frac{\text { bad pipes incorrectly classified as good }}{\text { number of pipes actually in bad condition }}
\end{aligned}
$$

The OSR indicates the predictive capabilities of the developed tree for the two pipe condition classes and the FNR indicates the tendency of the model to misclassify poor condition pipes. A high OSR in combination with a low FNR in a stratified test set ensures accurate and reliable predictions.

Each prediction made by the classification tree is associated with a propensity score. If a prediction of poor condition is made, propensity is the probability of predicting poor at the leaf node $(P)$ and is determined by the distribution of instances at the leaf (i.e. if $70 \%$ of the instances that make it to the leaf are poor and only $30 \%$ are good, then $P=0.70$ ). If the predicted condition at the leaf is good, then propensity is $1-P$.

Data mining algorithms tend to be most effective when there are equal numbers of instances in both classes. Any imbalance in the training set of instances can have a negative impact on the predictive performance for the minority class as the algorithm will tend to focus model building efforts on correctly classifying instances in the majority class. Instances in the minority class will usually be assigned to the majority class as the algorithm seeks to minimize the total number of misclassification errors. As an example, consider a dataset made up of $80 \%$ good pipes and $20 \%$ poor pipes. The data mining algorithm might choose to assign every pipe to the good class as this would achieve an OSR of $80 \%$. Assigning every pipe to the majority class would mean every poor pipe was incorrectly classified and this would be of limited utility when planning future inspections and understanding stormwater pipe deterioration.

One approach to dealing with data imbalance is to use the cost senstive learning capabilities of the C5.0 system. The C5.0 system treats misclassification costs as being equal using the default cost matrix (Original) shown in Table 4. The combination of imbalanced data and equal misclassification costs will result in a classification tree that focuses on correctly predicting good pipes at the expense of misclassifying poor pipes. The cost matrix 
can be adjusted to force the algorithm to pay greater attention to the classification of the poor pipes in the minority class. If, for example, the cost matrix (Modified) in Table 4 is used, misclassified poor pipes cost twice as much as misclassified good pipes and the 2:1 cost ratio changes the probability threshold at each leaf from 0.50 to 0.33 . With this cost ratio, a distribution of $60 \%$ good and $40 \%$ poor pipes at a leaf node will result in a classification of poor (as the algorithm wants to make the less costly decision when the distribution of instances are approximately equal).

As the true cost of stormwater pipe condition misclassification are not explicitly known, a variety of cost ratios can be evaluated. The C5.0 system takes this modified cost matrix into account when constructing the tree so that expected misclassification costs are minimized (IBM 2011).

Table 4 Modified cost matrix.

\begin{tabular}{lcllcc}
\hline & \multicolumn{2}{c}{ Original } & \multicolumn{2}{c}{ Modified } \\
Actual & Predicted Condition & Actual & \multicolumn{2}{c}{ Predicted Condition } \\
Condition & Poor & Good & Condition & Poor & Good \\
\hline Poor & 0 & 1 & Poor & 0 & 2 \\
Good & 1 & 0 & Good & 1 & 0 \\
\hline Note: The true costs of misclassification are unknown so a variety of cost ratios can be evaluated \\
when developing the classification tree.
\end{tabular}

\section{Results}

A classification tree predictive model developed without taking into account imbalance in the training set of instances had an OSR of $63 \%$ (268 out of 425 pipes correctly classified) and a FNR of $53 \%$ when predicting conditions in the stratified test set. The algorithm focused more on accurately classifying good pipes than it did on the poor pipes in the minority class. Its poor predictive performance made it unsuitable as a tool for planning future inspections.

The classification tree developed using the 2:1 modified cost ratio shown in Table 4 is shown in Figure 3. Classification results for the training and stratified test set are shown in Table 5. With a stratified test set OSR of $71 \%$ (301 out of 425 pipes correctly classified) and an FNR of $37 \%$ the classification tree represents a considerable improvement on the one constructed without accounting for data imbalance. The 2:1 cost ratio proved to be the most effective as larger ratios resulted in poor overall performance for both condition classes (e.g. 3:1 cost ratio resulted in an OSR of $61 \%$ with 263 out of 425 pipes in the test set correctly classified).

Table 5 Results for the classification tree.

\begin{tabular}{lcclcc}
\hline & \multicolumn{2}{c}{ Training } & \multicolumn{3}{c}{ Testing } \\
Actual & \multicolumn{2}{c}{ Predicted Condition } & Actual & \multicolumn{2}{c}{ Predicted Condition } \\
Condition & Poor & Good & Condition & Poor & Good \\
\hline Poor & 262 & 154 & Poor & 87 & 52 \\
Good & 217 & 641 & Good & 72 & 214 \\
\hline Accuracy $=903 / 1274=0.71$ & & Accuracy $=301 / 425=0.71$ \\
FNR $=154 / 416=0.37$ & & FNR $=52 / 139=0.37$
\end{tabular}

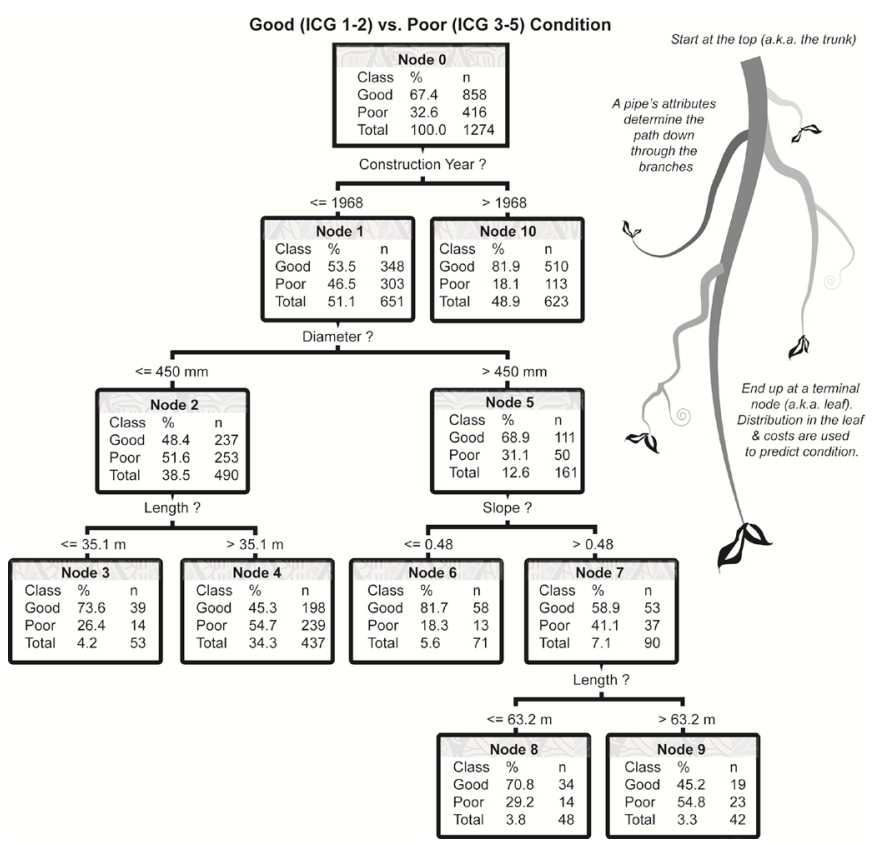

Figure 3 Stormwater pipe condition classification tree.

Each split in the tree provides insight into the influence of pipe-specific attributes on stormwater pipe structural conditions. The first split divides pipes according to their construction year, where $82 \%$ of the stormwater pipes placed after 1968 are in good condition. Pipes placed prior to 1968 are rapidly approaching or have already passed 50 y operation and $47 \%$ of them are now in poor structural condition (node 1). Although some pipes placed after 1968 are structurally unsound, $82 \%$ of these newer pipes are still in good structural condition (node 10).

A subsequent split divides older pipes into those with diameter $\geq 450 \mathrm{~mm}$ and those with diameter $<450 \mathrm{~mm}$. Approximately half of the older, small diameter stormwater pipes in Guelph are in poor structural condition (node 2). Older, large diameter pipes on the other hand are predominantly in good condition (node 5). The influence of diameter on pipe condition has been previously identified in the literature. Micevski et al. (2002) found that smaller diameter stormwater pipes in Australia are aging faster than bigger diameter pipes as designers often underestimate the traffic loads and cover requirements for small pipes. Davies et al. (2001) note that large diameter pipes are often put in place by more experienced personnel, reducing the likelihood for damage to occur during the installation process. While older, small diameter pipes have a $55 \%$ chance of being in poor condition if their lengths are greater than $35 \mathrm{~m}$ (node 4), shorter pipes have only a $26 \%$ chance of being in poor condition (node 3 ). This is likely caused by an increased number of joints in longer pipes leading to more possible points of failure than in shorter pipes.

Pipes constructed prior to 1968 with diameter $>450 \mathrm{~mm}$ and steeper slopes are more likely to be in poor condition than those with flatter slopes (nodes 6 and 7). Pipe sections with 
steeper slopes tend to have the highest deterioration rates as flow rates are generally faster in steep sections. This creates a greater possibility for erosion and surface wear of the wall inside of the pipe (Baik et al. 2006). The influence of length on pipe condition is once again apparent in nodes 8 and 9, where $55 \%$ of those pipes with length $>63 \mathrm{~m}$ are in poor condition, compared to only $29 \%$ of those with a shorter length.

The classification tree does not provide an indication of the influence of material on condition, as splitting the instances by that attribute did not improve predictive performances. Of the 651 pipes in the training set constructed prior to 1968 (node 1), 627 are made of concrete and division according to pipe material is therefore unnecessary. Although there is a more uniform distribution of material for pipes constructed after 1968 (168 concrete, 224 extra strength concrete and 231 reinforced concrete), any tree branches incorporating material on the right side of the tree were pruned away by the C5.0 system as they did not improve predictive performance. For purposes of knowledge discovery, the modeling software can be used to drill down into any relationship between material and condition for these newer pipes in the training set. As illustrated in Figure 4, very few of the inspected reinforced concrete pipes were found to be in poor structural condition.

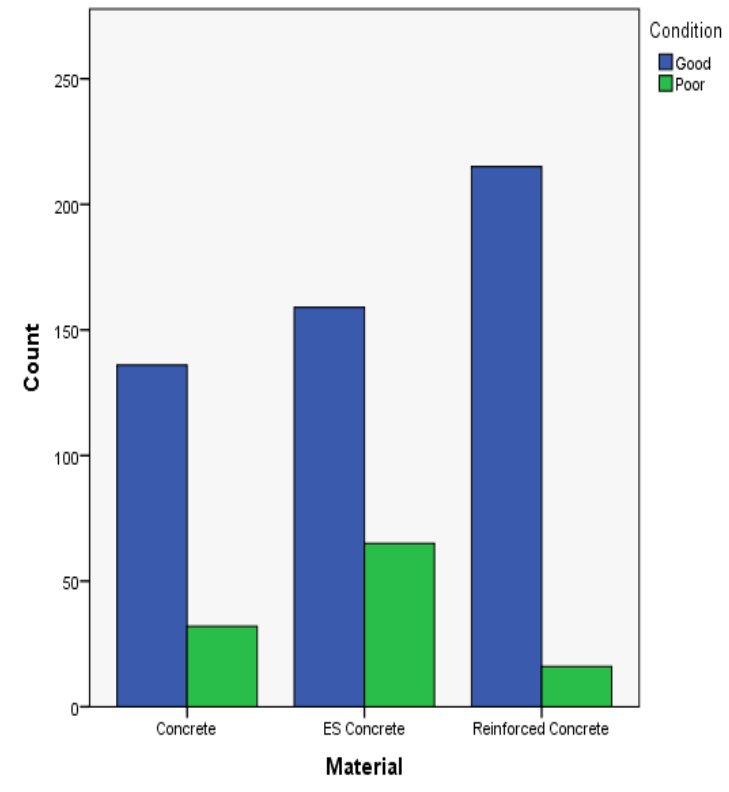

Figure 4 Distribution of condition for pipes constructed after 1968.

\section{Discussion}

\subsection{Planning Future Inspections}

The developed classification tree can be used to predict the condition of the 1596 concrete, 422 extra strength concrete and 1764 reinforced concrete pipes that have not been inspected. The tree predicts the large majority of these pipes are in good condition as 3360 pipes were constructed after 1968. Of these newer pipes, 2733 are $<25$ y old and are likely of limited threat to the overall integrity of the stormwater network. Resources for future inspection should instead be dedicated to the 265 uninspected pipes the tree classifies (predicts) as being in poor structural condition.

A total of 859 uninspected pipes have an unspecified material type listed in the construction records. These can still be presented to the classification tree to gain a general idea of their potential condition. The tree classifies 247 out of the 859 unspecified material pipes as being in poor condition. These 247 pipes can be added to the 265 pipes previously designated for future condition inspection.

A total of 941 uninspected pipes are made from a variety of materials other than concrete and cannot be presented to the classification tree. Of these pipes:

- 466 are made of PVC and are <25 y old;

- 161 pipes are made of corrugated steel, of which 93 have diameter $>1 \mathrm{~m}$; and

- 311 pipes are made from materials such as asbestos cement and corrugated metal pipe, of which 243 are $<25$ y old.

It is up to the discretion of utility managers to add any of these 941 pipes to the list of pipes that are targeted for immediate inspection.

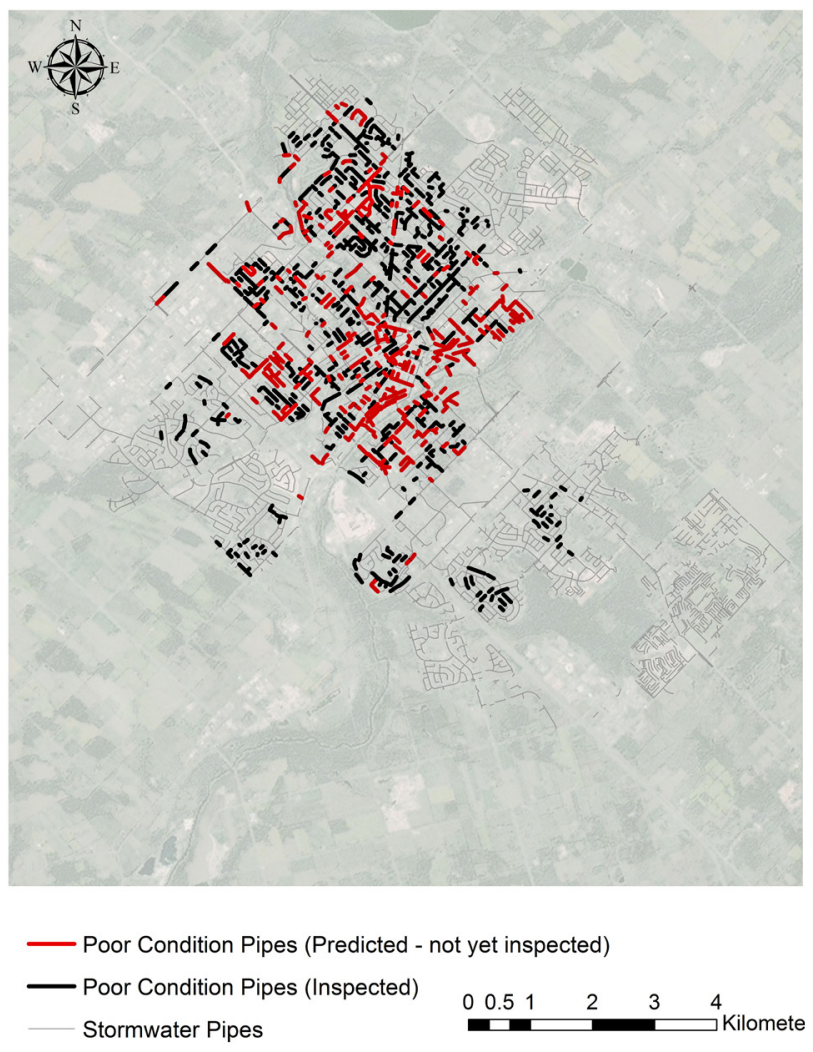

Figure 5 Location of poor condition stormwater pipes.

A total of 2458 out of 8073 stormwater pipes were inspected from 2008 to 2011 . Of these inspected pipes, 901 were 
found to be in poor condition. The classification tree identified an additional 512 pipes that have not been inspected but are likely to be in poor condition. The location of the 1413 (901 inspected, 512 predicted) poor condition pipes can be visualized using ESRI ArcMap (Figure 5).

\subsection{Improving Predictive Performance}

The dataset available for model development lacked information on burial depth and this may have negatively impacted the predictive performance of the developed models as the influence of depth pipe condition has been identified in the literature. Lester and Farrar (1979) note that the frequency of (sewer) pipe defects decreases as burial depths increase. Shallow sewers failed faster than deeper pipes in Cullen (1982). Obtaining reliable data on soil conditions around each pipe would likely improve the predictive capabilities of the classification tree. Soil type determines the rate of infiltration around the pipe and the concrete corrosion potential. The dataset available for model development was imbalanced, with more good condition pipes than poor. Future inspection of pipes predicted by the classification tree to be in poor condition would restore balance to the dataset available for model development and may improve predictive performance.

\section{Conclusion}

CCTV inspections are commonly used to determine the condition of aging stormwater pipes. This work is expensive and most municipalities are limiting their inspections to small portions of their stormwater network. Data mining with classification trees enhanced the value of existing inspection records by gaining insight into the stormwater pipe deterioration process.

A classification tree was developed using 107 km inspection data obtained in Guelph, Ontario (representing one third of the stormwater network). The classification tree clearly illustrates the influence of construction year, diameter, length and slope on pipe condition. Year of construction was found to strongly influence stormwater pipe structural condition, with $82 \%$ of those pipes placed after 1968 in good structural condition and 45\% of those placed prior to 1968 in poor structural condition. Pipe diameter, slope and length were found to strongly influence the resulting condition of the older pipes in the stormwater system.

The tree reliably predicts pipe condition (with 301 out of 425 correctly classified instances in a stratified test set) and can therefore be used to identify the location of structurally unsound pipes that have not yet been inspected. The tree predicts 512 pipes that have not yet been inspected are likely in poor structural condition. These pipes should be targeted for the next round of inspection as there is a basis to assess their threat to the integrity of the stormwater network.

Data mining provides an opportunity to go beyond simply inspecting pipes and assigning condition grades. Classification trees are a logical choice for extracting information from inspection datasets as they are straightforward in their implementation and are easy to understand. Any municipality with a pipe inspection dataset can adopt the presented methodology to develop their own powerful classification tree models. The combination of inspection and data mining can guide future inspections and will be valuable when dealing escalating levels of stormwater pipe deterioration.

\section{Acknowledgments}

The authors would like to thank the City of Guelph for the provision of the stormwater inspection records and assistance throughout the research process. Thank you to the Data Resources Centre at the University of Guelph for their assistance with mapping and GIS analysis. This research was funded by the University of Guelph, the Natural Sciences and Engineering Research Council of Canada and the Canada Research Chairs program.

\section{References}

Ana, E. and W. Bauwens. 2010. "Modeling the Structural Deterioration of Urban Drainage Pipes: The State-Of-The-Art in Statistical Methods." Urban Water Journal 7 (1): 47-59. doi: 10.1080/15730620903447597.

Baik, H., H. Jeong and D. Abraham. 2006. “Estimating Transition Probabilities in Markov Chain-Based Deterioration Models for Management of Wastewater Systems." Journal of Water Resources Planning and Management 132 (1): 15-24.

Berry, M. and G. Linoff. 2004. Data Mining Techniques: For Marketing, Sales and Customer Relationship Management, 2nd ed. Indianapolis: Wiley.

CIRP. 2012. “Canadian Infrastructure Report Card: Municipal Roads and Water Systems." Westmount, Quebec: Canadian Society for Civil Engineering. http://www.canadainfrastructure. $\mathrm{ca} / \mathrm{en} /$.

Cullen, N. 1982. "The Sewer Deterioration Problem: Evidence from Collapse Studies." Chap. 2 in Restoration of Sewerage Systems. London: Thomas Telford/ICE Publishing. ISBN 9780-7277-4911-6.

Davies, J., B. Clarke, J. Whiter and R. Cunningham. 2001. “Factors Influencing the Structural Deterioration and Collapse of Rigid Sewer Pipes." Urban Water 3 (4): 277-86.

Holtz, S. 2006. There Is No "Away"_-Pharmaceuticals, Personal Care Products and Endocrine-Disrupting Substances: Emerging Contaminants Detected in Water. Toronto: Canadian Institute for Environmental Law and Policy.

Hurley, M. 2012. "Sinkhole Swallows Car, Closes Eastbound Highway 174." Ottawa: The Ottawa Citizen, 20120905.

IBM. 2011. IBM SPSS Modeler 14.2: Modeling Nodes. Armonk, NY: IBM Corporation.

Karpf, C. and P. Krebs. 2011. “Quantification of Groundwater Infiltration and Surface Water Inflows in Urban Sewer Networks Based on a Multiple Model Approach." Water Research 45 (10): 3129-36. 
Kessler, R. 2011. “Stormwater Strategies: Cities Prepare Aging Infrastructure for Climate Change." Environmental Health Perspectives 119 (12): a514-20. doi:10.1289/ehp.119-a514.

Kolpin, D., E. Furlong, M. Meyer, E. Thurman, S. Zaugg, L. Barber and H. Buxton. 2002. "Pharmacueticals, Hormones and Other Organic Wastewater Contaminants in U. S. Streams 1999-2000: A National Reconniassance." Environmental Science and Technology 36:1202-11.

Lester, J. and D. Farrar. 1979. An Examination of the Defects Observed in Six Kilometers of Sewers. Wokingham, Berkshire: Transport Research Laboratory. TRRL Supplemtary Report 531.

McCarthy, K., B. Zabar and G. Weiss. 2005. "Does Cost-Sensitive Learning Beat Sampling for Classifying Rare Classes?" In UBDM '05: Proceedings of the First International Workshop on Utility-Based Data Mining, 69-77. New York: ACM (Association for Computing Machinery). doi:10.1145/1089827.1089836.

Micevski, K., G. Kuczera and P. Coombes. 2002. "Markov Model for Stormwater Pipe Deterioration." Journal of Infrastructure Systems 8 (2): 49-56.

Moore, M. and J. Carpenter. 2010. "A Decision Tree Approach to Modeling the Private Label Apparel Consumer." Marketing Intelligence and Planning 28 (1): 59-69.

Podgorelec, V., P. Kokol, B. Stiglic and I. Rozman. 2002. “Decision Trees: An Overview and their Use in Medicine." Journal of Medical Systems 26 (5): 445-63.

Quinlan, J. 1993. C4.5: Programs for Machine Learning. Burlington, MA: Morgan Kaufmann.

Quinlan, J. 2012. C5.0: An Informal Tutorial. Rulecrest Research. http://www.rulequest.com.

Reutter, M. 2012. "Millions more to be sunk into Monument Street sinkhole." Baltimore, MD: Baltimore Brew, 20121109.

Rokach, L. and O. Maimon. 2007. Data Mining with Decision Trees: Theory and Applications. Singapore: World Scientific Publishing.

\section{R. Richard Harvey University of Guelph, Guelph, Ontario.}

Edward A. McBean University of Guelph, Guelph, Ontario.
Sahin, Y. and E. Duman. 2011. "Detecting Credit Card Fraud by Decision Trees and Support Vector Machines." In Proceedings of the International Multi-Conference of Engineers and Computer Scientists, Vol. 1, 1-6. IAENG (International Association of Engineers). http://www.iaeng.org.

Sauer, E., J. Vanderwalle, M. Bootsma and S. McLellan. 2011. “Detection of the Human Specific Bacteroides Genetic Marker Provides Evidence of Widespread Sewage Contamination of Stormwater in the Urban Environment." Water Research 45 (14): 4081-91. doi:10.1016/j.watres.2011.04.049.

Sercu, B., L. van de Werfhorst, J. Murray and P. Holden. 2011. "Sewage Exfiltration as a Source of Storm Drain Contamination during Dry Weather in Urban Watersheds." Environmental Science and Technology 45 (17): 7151-7. doi:10.1021/es200981k.

Tooke, T., N. Coops, N. Goodwin and J. Voogt. 2009. “Extracting Urban Vegeatation Characteristics Using Spectral Mixture Analysts and Decision Tree Classifications." Remote Sensing of Environment 113 (2): 398-407.

Tran, H. 2007. Investigation of Deterioration Models for Stormwater Pipe Systems. Melbourne: Victoria University. PhD Dissertation.

Tran, H. and A. Ng. 2010. "Classifying Structural Condition Of Deteriorating Stormwater Pipes Using Support Vector Machine." In Pipelines 2010: Climbing New Peaks to Infrastructure Reliability, edited by G. Ruchti and T. Roode, 857-66. Reston, VA: ASCE (American Society of Civil Engineers). doi: $10.1061 / 41138(386) 82$.

USEPA. 2009. National Water Quality Inventory: Report to Congress, 2004 Reporting Cycle. Washington, DC: U. S. Environmental Protection Agency. EPA 841-R-08-001.

Witten, I., E. Frank and M. Hall. 2011. Data Mining: Practical Machine Learning Tools and Techniques, 3rd ed. Burlington, MA: Morgan Kaufmann. 